\title{
GEOGRAFIA DA SAÚDE: UM ESTUDO SOBRE A MALÁRIA NA ZONA URBANA DE SÃO JOÃO DA BALIZA-RORAIMA
}

\author{
Pedro Rufino Silva \\ Graduação em Licenciatura em Geografia - UERR \\ Técnico em Entomologia - SESAU \\ prsbv@hotmail.com
}

क.

Marcelo Santos da Silva

Professor Mestre da Universidade Estadual de Roraima - UERR

natal.boavista@gmail.com

\section{RESUMO}

Situado na Amazônia brasileira, o estado de Roraima possui extensas áreas maláricas, localizadas principalmente em regiões com predominância de Floresta Tropical densa. Em regiões onde predomina o Cerrado (lavrado roraimense), a endemia se concentra nas proximidades de rios e igarapés com vegetação ciliar de médio e grande porte. Dentre os municípios de Roraima que apresentam maior número de casos de malária, São João da Baliza concentra a maior incidência de casos urbanos da doença. 0 presente trabalho tem como objetivo descrever os aspectos ambientais e socioeconômicos do bairro Universo na cidade de São João da Baliza que contribuem com a dinâmica espacial de transmissão da malária. Tem como metodologia um estudo descritivo com dados levantados mediante a consulta no banco de dados informatizado do SIVEP-Malária e visitas de campo. A partir da análise dos dados, verificou-se que essa localidade possui uma rica bacia hidrográfica formada por igarapés que são o principal sítio de reprodução do An. (Ny) darlingi, principal vetor da malária na Amazônia e ocupada por habitações localizadas nas proximidades do ambiente de reprodução dos mosquitos da malária. Imóveis que não impedem o contato do homem com o vetor. Com cerca de $20 \%$ da população urbana da cidade de São João da Baliza, o bairro Universo contribuiu com $29,47 \%$ dos casos de malária que corresponderam ao IPA de 264 casos para 1000 habitantes em 2014. Foi possivel constatar que os fatores ambientais e socioeconômicos exercem uma relação determinante para a ocorrência de casos de malária urbana no bairro Universo. Os resultados da análise dos fatores mencionados irão contribuir para um planejamento mais criterioso das ações de controle vetorial tornando-as mais efetivas.

Palavras - chave: Malária urbana; Fatores ambientais; Socioeconômico; Controle vetorial.
Located in the Brazilian Amazon, the state of Roraima has extensive malarious areas, mainly located in regions with dense tropical forest predominance. In regions where the Cerrado predominates (roraimense), the endemic is concentrated in the vicinity of rivers and streams with medium and large ciliary vegetation. Among the municipalities of Roraima that present the highest number of cases of malaria, São João da Baliza concentrates the highest incidence of urban cases of the disease. The present work aims to describe the environmental and socioeconomic aspects of the neighborhood Universo in the city of São João da Baliza that contribute to the spatial dynamics of malaria transmission. Its methodology is a descriptive study with data collected through consultation in the SIVEP-Malaria computerized database and field visits. From the analysis of the data, it was verified that this locality has a rich hydrographic basin formed by igarapés that are the main reproduction site of An. (Ny) darlingi, the main vector of malaria in the Amazon and occupied by dwellings located in the vicinity of environment of malaria mosquitoes. Properties that do not impede the contact of the man with the vector. With around $20 \%$ of the urban population in the city of São João da Baliza, the Universo neighborhood contributed $29.47 \%$ of malaria cases that corresponded to the IPA from 264 cases to 1000 inhabitants in 2014. It was possible to verify that environmental and socioeconomic variables have a determinant relationship for the occurrence of urban malaria cases in the Universo neighborhood. The results of the analysis of the mentioned factors will contribute to a more judicious planning of vector control actions, making them more effective.

Key - words: Urban malaria; Environmental factors; Socioeconomic; Vector control. 


\section{INTRODUÇÃO}

Este artigo é um recorte do Trabalho de Conclusão de Curso (TCC) apresentado ao Curso de Licenciatura em Geografia da Universidade Estadual de Roraima, no ano de 2015. O estudo buscou dimensionar quais os fatores ambientais e socioeconômicos envolvidos na dinâmica de transmissão da malária urbana na cidade de São João da Baliza, destacando o papel da Geografia da Saúde e o Sistema de Informação Geográfica (SIG) no entendimento das relações entre saúde e ambiente, facilitando a análise das características epidemiológicas das áreas que favorecem a concentração de agravos à saúde e, apontar procedimentos que possam contribuir para tomada de decisão por parte dos gestores municipais nas ações de prevenção e controle da malária.

Situado na Amazônia brasileira, o estado de Roraima possui extensas áreas maláricas, localizadas principalmente em regiões com predominância de floresta tropical densa. Nas regiões onde predomina a paisagem savânica (denominadas popularmente de lavrado pelos roraimenses), a endemia se concentra de forma focal em áreas com vegetação ciliar de médio e grande porte, situadas principalmente, nas margens de rios e igarapés. Essas regiões apresentam todos os fatores ambientais favoráveis para consolidação da cadeia de transmissão da doença tais como, rica bacia hidrográfica com abundante vegetação ciliar, índices de precipitação e umidade elevados, fluxo migratório, presença do vetor transmissor e de hospedeiro humano susceptível ao parasita, agente causador da malária.

Neste contexto, o município de São João da Baliza (SJB) situa-se ao sul do estado de Roraima e apresenta uma região de floresta tropical densa, endêmica para malária. A extensão períurbana da sede do município tem o seu entorno periférico próximo a área florestal. Essa característica, associada com a localização de diversos cursos naturais de água na área urbana do município, faz dessa localidade uma área de risco para a transmissão da malária. Nos últimos anos, os altos índices de casos da doença no perímetro urbana vêm se manifestando de forma constante.

A incidência de casos de malária urbana na referida cidade está estreitamente relacionada com os determinantes ambientais e socioeconômicos, pois as áreas localizadas próximas à vegetação ciliar de igarapés que compõem a bacia hidrográfica da cidade de São João da Baliza apresentam todas as condicionantes ambientais favoráveis para o desenvolvimento dos vetores da malária. Assim, a população residente nessas áreas situada próximas coleções hídricas, sítios de reprodução dos vetores da malária, facilita o contato homem/vetor, um fator crucial para consolidar o ciclo de transmissão da doença.

Dimensionar qual a relação dos fatores determinantes para a ocorrência de casos de malária urbana em SJB é fundamental como aporte auxiliar no processo de controle da endemia. Para tanto, a Geografia da Saúde e o Sistema de Informação Geográfica (SIG) podem contribuir para o entendimento das relações entre saúde e ambiente, facilitando a análise das características epidemiológicas das áreas que favorecem a concentração de agravos à saúde.

Contudo, este trabalho teve como objetivo apresentar os aspectos ambientais e socioeconômicos do bairro Universo, da cidade de São João da Baliza, contribuindo com a dinâmica espacial de transmissão da malária na ótica da geografia da saúde; relacionar os fatores descritos na dinâmica espacial de ocorrência dos casos da doença na área de estudos e apontar procedimentos que possam contribuir para tomada de decisão por parte dos gestores municipais nas ações de prevenção e controle desse agravo.

\section{METODOLOGIA}


O presente estudo tem como proposta de pesquisa a óptica teórica com procedimentos de estudo de caso e pesquisa documental, levando a um objetivo descritivo para dimensionar a relação dos fatores ambientaise socioeconômicos relacionados com a dinâmica espacial da ocorrência de casos de malária urbana condicionando a uma abordagem quali-quantitativa. Segundo Gil (2009, p. 42) a pesquisa descritiva "[...] têm como objetivo primordial a descrição das características de determinada população ou fenômeno, ou então o estabelecimento de relações entre as variáveis". Corroborando, Gonçalves (2007, p. 67) esse método de pesquisa "[...] objetiva descrever as características do objeto de estudo".

$\mathrm{Na}$ descrição das características ambientais e o desenvolvimento da pesquisa, foram realizadas visitas ao bairro Universo, delimitado como área de estudo. Já nas análises dos dados epidemiológicos dos casos de malária (série histórica de 2004 a 2014) estabeleceram-se a compreensão da interação da doença com o ambiente e o homem.

Portanto, para a definição do local de estudo, levou-se em consideração os índices elevados de casos de malária urbana registrados no bairro, os fatores ambientais favoráveis para a reprodução dos Anofelinos, vetores transmissores da doença e os fatores socioeconômicos, por se tratar de uma área periférica constituída por habitações localizadas nas proximidades do ambiente de reprodução dos mosquitos da malária.

\section{Procedimentos de Coleta de Dados}

Os dados foram levantados a partir de consulta no banco de dados informatizado do "Sistema de Informação de Vigilância Epidemiológica - Notificação de Casos de Malária" (SIVEP-Malária), gerenciado pela Secretaria de Vigilância em Saúde (SVS) do
Ministério da Saúde (MS), disponibilizados "online". Período consultado - 2004 a 2014, e pesquisas de campo realizadas para observação “in loco" das feições ambientais, aspectos dos imóveis e produção de imagens terrestres e aéreas com câmera digital e Drone Dji Phantom com câmera acoplada.

\section{Análises dos Dados}

Os procedimentos foram realizados a partir da análise do material produzido pelo levantamento epidemiológico, caracterização da área de estudo (bairro Universo) e questionários aplicados aos moradores para dimensionar a correlação da autoctonia dos casos de malária com os fatores ambientais, socioeconômicos e culturais dessa localidade. Considerando que para a ocorrência de determinados agravos envolvendo a dinâmica espacial de transmissão da malária, tais fatores são imprescindíveis para a consolidação desses agravos.

Para uma melhor compreensão, os dados foram tabulados e analisados com auxílio da planilha Excel Microsoft Office 2010 e com o Software QGIS, programa de Sistema de Informação Geográfica com código aberto de Licença Pública Geral GNU, disponível no sítio: http://qgis.org/pt_BR/site/forusers/ download.html.

\section{Caracterização da área de estudo}

A presente pesquisa foi realizada no município de São João da Baliza, localizado na região sudeste do Estado de Roraima. A extensão territorial do município é de 4.284,507 km2 e a densidade demográfica, de 1,58 hab/ $\mathrm{km}^{2}$, encontra-se a $0^{\circ} 56^{\prime} 58^{\prime \prime}$ de latitude Norte e 59 54' 41" de longitude Oeste. A altitude é de $117 \mathrm{~m}$ acima do nível do mar, tem como limites: ao norte, município de Caracaraí; ao sul, estado do Amazonas; ao leste, município de 
Caroebe; ao oeste, municípios de Rorainópolis e São Luiz do Anauá (BRASIL, 2010).

A área escolhida como objeto de estudo foi o bairro Universo, localizado na porção Norte da sede do município de São João da Baliza (Mapa 01). Essa localidade, de acordo com os dados do SIVEP-Malária, tem uma população estimada de 908 habitantes o que corresponde a cerca de $20 \%$ da população urbana do município. O bairro Universo está inserido numa região composta por corpos hídricos formados por igarapés com abundante vegetação ciliar que favorece a reprodução dos vetores da malária.

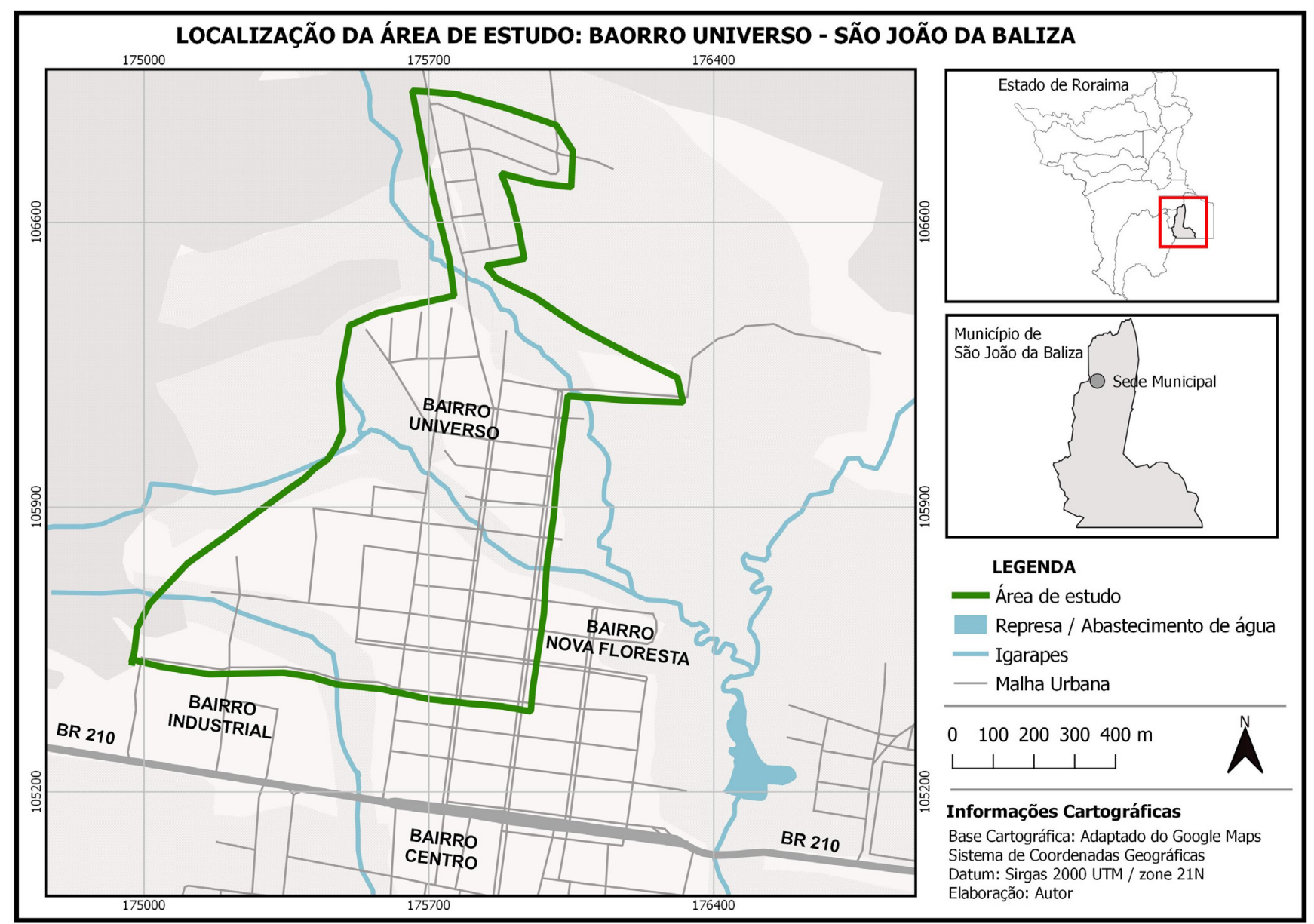

Mapa 01 - Mapa com a localização da área de estudo

Fonte: Autor, 2019.

\section{RESULTADOS E DISCUSSÕES}

\section{Breve histórico da Geografia da Saúde}

Inicialmente surgiu a Geografia Médica, para posteriormente ser denominada Geografia da Saúde. Pertence a Hipócrates, o pai da Medicina, o grande legado de romper, ainda no século $\mathrm{V}$ a. $\mathrm{C}$, com o paradigma da explicação divina para a origem das doenças e explicá-las através do estudo das características físicas do ambiente. Acreditase que foi nessa época, que surgiu a Geografia da Saúde que passou a utilizar os saberes da Medicina eda Geografia, constituindo-seem um campo interdisciplinar de pesquisa. É possivel afirmar que este campo do conhecimento tem uma história longa e evoluiu sob nomes variados como, "topografia médica", "geografia patológica", "epidemiologia geográfica", 
"geomedicina", "geografia médica", dentre outros (RODRIGUES, 2004).

Pessoa (1978, p. 87) ao definir Geografia médica diz que,

A Geografia Médica tem por fim o estudo da distribuição e da prevalência das doenças na superficie da terra, bem como de todas as modificações que nelas possam advir por influência dos mais variados fatores geográficos e humanos.

Neste contexto, a Geografia médica pode contribuir com a análise de fatores ecológicos e epidemiológicos decorrentes da interação do homem com o ambiente, que passam a existir a partir do processo de ocupação do espaço pelo homem, e contribuem para o surgimento de agravos e patologias num determinado território.

Para Lacaz, Baruzzi e Siqueira Junior (1972, p. 1),

Geografia Médica é a disciplina que estuda a geografia das doenças, isto é, a patologia à luz dos conhecimentos geográficos. Conhecida também como Patologia geográfica, Geopatologia ou Medicina geográfica, ela se constitui em um ramo da Geografia humana (Antropogeografia) ou, então, da Biogeografia.

A Geografia da Saúde tem como principal função contribuir com a análise do espaço considerando suas feições e dinâmicas para ampliação de conhecimento sobre o processo de ocupação e a sua influência no padrão de saúde de uma localidade. Esse processo se materializa na adoção de ações de caracterização de ambientes modificados que potencializam surtos epidémicos e tem como finalidade aperfeiçoar atividades de prevenção e combate a esses agravos na área da saúde, utilizando ferramentas como, por exemplo, o sensoriamento remoto e o geoprocessamento que contribuem no monitoramento e vigilância do território a ser analisado (GALLO e CARVALHO, 2011).

As análises espaciais voltadas para as questões de saúde foram impulsionadas no início da dominação europeia no novo mundo, quando se desenvolveu a maior fase de produção da Geografia da Saúde. Havia, naquela época, uma grande preocupação em conhecer as doenças mais frequentes que ocorriam nas regiões conquistadas, principalmente nas de clima tropical (RODRIGUES, 2004).

Foi a partir da década de 1950 que se iniciaram no Brasil as primeiras pesquisas voltadas para as doenças presentes nas áreas que estavam passando pelo processo de interiorização e integração do território brasileiro, em meio a esse processo, a Geografia da Saúde no Brasil iniciava seus primeiros estudos. Antes já havia adotado mudanças fundamentais necessárias para o arcabouço teórico-metodológico para o qual se propunha (ALIEVI e PINESE, 2013).

Na opinião de Perehouskei e Benaduce (2007), a realização dos estudos em Geografia Médica voltados para a melhoria das condições de saúde da sociedade se intensificaram na década 1982, a partir de discussões em eventos acerca da Geografia da Saúde, como o Congresso da União Geográfica Internacional (UGI) onde se definiu a mudança do termo Geografia Médica para Geografia da Saúde.

De acordo com Santos (2010, p. 49) “a Geografia da Saúde pode ajudar nos problemas referentes à saúde, se tiver como eixo norteador o ambiente, a sociedade e o território". O autor também menciona que essa nova denominação de Geografia da Saúde é mais sistêmica, preocupada não apenas com o enfermo, mas também com o que está por trás dessa enfermidade e a qual situação socioeconômica e ambiental determinado indivíduo está sujeito diariamente.

Diante do exposto, é possivel afirmar que a Geografia da Saúde é uma ferramenta de grande valia para os programas de saúde, 
pela sua contribuição nas análises espaciais de ambientes modificados. Para Perehouskei e Benaduce (2007, p. 37) “a Geografia da Saúde apresenta linhas de pesquisa, voltadas tanto para a Geografia Física, como para a Geografia Humana, ambas utilizam os Sistemas de Informações Geográficas - SIGs na maioria dos estudos desenvolvidos na área de saúde". Portanto, a geografia da saúde e os SIGs podem fornecer subsídios seguros ao setor de Epidemiologia, para que este possa elaborar programas de vigilância ambiental tanto no aspecto preventivo como no controle das endemias.

\section{Malária, um breve histórico}

Definir a origem da malária no planeta não é uma tarefa simples. Diante da escassez de informação e da fragmentação dos estudos para determinar a real origem da doença, é possível admitir que a malária tenha se originado na África tropical onde o parasita, ao passar por um longo processo de adaptação, passou a se desenvolver em hospedeiros, inclusive em humanos (BRASIL, 2006).

Para Silva (2011, p. 28) "a exemplo do que ocorre atualmente, o homem passou a ser um hospedeiro ocasional ao ocupar os espaços onde antes existiam apenas os animais alvos preferidos dos mosquitos transmissores da malária".

Em relação à incidência de casos da doença em humanos, pesquisas evidenciaram que a malária assola a humanidade desde a mais remota antiguidade. "A prova disso está evidenciada em estudos arqueológicos que demonstram a doença por meio de relatos de febre [...], além de inscrições em templos egípcios descrevendo casos de febre intermitente" (BRASIL, 2006, p. 21).

Há evidências que na Grécia, no século IV a.C., a malária foi endêmica. Coube a Hipócrates (460-377 a.C.) a primeira rejeição ao misticismo atribuído à causa da doença, imposta como um castigo dos deuses. Hipócrates documentou os casos febris característicos da malária e os relacionou às proximidades dos indivíduos com as coleções de água estagnadas (FRANÇA et al., 2008 apud SILVA, 2011). Segundo o autor, foi essa ligação da febre com áreas alagadas que, séculos mais tarde, exatamente no século XIV, os italianos passaram a chamá-la de mal aria (mau ar), por acreditarem que a doença era transmitida pelo odor fétido que emanava dos pântanos.

Quanto à origem da malária no novo mundo, esta ainda permanece sob questionamento. 0 que existe, portanto, de mais consistente é que essa doença era totalmente desconhecida pelas populações das novas terras, como relatam os documentos coloniais. Contudo, há evidências que foi a partir do contato com o conquistador europeu tanto na América do Norte, quanto na Central e do Sul que os casos de malária sugiram nas Américas (KIPLE, 1993 apud SILVA, 2011).

Segundo Silva (2011, p. 30) “à medida que as colônias avançavam em direção ao interior da América do Norte a malária assentava-se junto". O autor também relata que o avanço da malária no novo continente teve como dois dos principais limitadores a latitude e a longitude, as quais regulavam a proliferação dos vetores. Ao passo em que outras regiões com condições geográficas mais favoráveis a doença tornava-se endêmica.

\section{Epidemiologia da malária}

A malária ainda está presente nos dias atuais em cerca de 90 países situados principalmente na faixa entre os trópicos do globo terrestre. De acordo com a Organização Mundial da Saúde (OMS), a malária é um grande problema de saúde pública nos países em desenvolvimento. Estima-se que a população infectada por malária no planeta seja da ordem de 300 a 500 milhões de pessoas a cada ano com $90 \%$ dos casos registrados nos países do continente africano (BRASIL, 2002).

Apesar dos números impressionantes, a 
malária hoje se concentra de forma focal na maior parte do mundo. Apenas determinadas regiões, em cada país, continuam apresentando transmissão natural da doença. A situação atual é completamente diferente daquela que existia antes da Campanha de Erradicação, patrocinada pela Organização Mundial da Saúde (OMS) com o apoio da Organización Panamericana de la Salud (OPAS) e do governo norte-americano (através do Ponto IV e da Aliança para o Progresso), a partir de 1960, em todos os continentes. Naquele momento, a malária se apresentava como "doença de massa", atingindo vastas áreas e grandes contingentes populacionais em cada um dos países, onde a transmissão era endêmica (BARATA, 1995).

A malária é uma parasitose provocada por protozoários do gênero Plasmodium com ciclos complexos de multiplicação sexuada e assexuada. São quatro as espécies de Plasmódios que infectam o homem: Plasmodium vivax, Plasmodium falciparum, Plasmodium malariae e Plasmodium ovale, este último restrito ao continente africano (SILVA e OLIVEIRA, 2002). A malária está presente mais intensamente nos países tropicais onde as condições climáticas são mais favoráveis à proliferação dos vetores transmissores da doença (SANTOS, 2009).

O processo de transmissão da doença ocorre de pessoa a pessoa pela picada de mosquitos do gênero Anopheles que se infectam ao sugar o sangue de um hospedeiro humano doente. Nas regiões equatoriais doenças como a malária são transmitidas de forma constante. Isso se deve ao favorecimento dos fatores climáticos (altos índices pluviométricos, temperatura e umidade relativa do ar elevadas). Nas áreas subtropicais, a temperatura cai nos meses de inverno, impedindo a atividade dos vetores. Nesse período, a transmissão da malária é interrompida pela baixa temperatura nos meses mais frios (LEMOS e LIMA, 2002).

Reconhecidamente a malária é um grave problema de saúde pública no mundo. Além dos problemas socioeconômicos gerados por essa doença, ela só é superada em números de mortes pela AIDS. Dentre as regiões globais mais afetadas pela malária as principais são: a África, o Sudeste Asiático e a América Latina (COELHO, 2010).

Para Brasil (2002, p. 14) "na região das Américas, o Brasil é o País que mais registra casos de malária, mais ou menos $50 \%$ dos casos, e a região amazônica é responsável pela quase totalidade dos casos de malária, cerca de 99,7\%". A doença divide o território brasileiro em duas áreas: a Região Endêmica, constituída pelos estados do Acre, Amapá, Amazonas, Maranhão, Mato Grosso, Pará, Rondônia, Roraima, Tocantins, e a Região NãoEndêmica, constituída pelos demais estados da federação (BRASIL, 2006). Para a determinação de áreas de risco de transmissão da malária no Brasil utiliza-se a Incidência Parasitária Anual (IPA) ${ }^{1}$, classificada em baixo risco, médio risco e alto risco.

Nas regiões consideradas en dêmicas para malária, a doença não é transmitida de maneira uniforme com igual rapidez ou intensidade. A dinâmica de transmissão (geralmente se dá de forma variável), dependente da interação de fatores ambientais, socioeconômicos, culturais e políticos. A junção desses fatores determina os níveis de riscos para contrair a doença, bem como a escolha das modalidades de controle a serem adotadas (BRASIL, 2006).

Localizado na Região Amazônica, o Estado de Roraima, com cerca de 225.000 km2 de superficie, apresenta a menor taxa brasileira de densidade demográfica (cerca de 1,5 hab/ $\mathrm{km} 2$ ). Contudo, quando se analisam os dados epidemiológicos, Roraima apresenta o maior número de casos de malária por habitante na Amazônia, apesar da ocorrência em seu território de apenas 5\% dos casos brasileiros da doença. Em 2000 foram registrados aproximadamente 110 casos para cada 1000

1 Baixo risco (IPA < 10/1.000 habitantes); Médio risco (IPA entre 10 - 49/1.000 habitantes); Alto risco (IPA $\geq$ $50 / 1.000$ habitantes) 
habitantes (BARATA, 1995, apud DIAS, 2003).

A malária é uma doença predominantemente rural. No entanto, na periferia de áreas urbanas da Amazônia, inclusive de grandes cidades, com características ambientais favoráveis para a reprodução dos vetores, tem havido transmissão da doença, decorrente da presença de portadores infectados provenientes da área rural, de localidades onde há circulação de plasmodium e a presença de vetores (BRASIL, 2010a). Na cidade de São João da Baliza, áreas com essas características de espaços rurais estão localizadas também no entorno da cidade, próximas de igarapés com abundante vegetação ciliar, desempenhando papel importante na reprodução dos vetores da malária.

A cidade de São João da Baliza está entre as localidades que apresentam maior incidência de casos de malária urbana no Estado de Roraima, sua localização está inserida numa região que apresenta todos os fatores ambientais necessários para a proliferação dos vetores da doença. Outro fator que também contribui para a transmissão da malária urbana em São João da Baliza é o seu ordenamento urbano. A produção de espaços próximos às margens de recursos hídricos, alterando o contexto paisagístico com a construção de aglomerados residenciais formados por imóveis cuja estrutura construtiva, na maioria dos casos, não impede o contato homem/vetor, favorece o ciclo de transmissão da doença nesses locais.

Conhecendo a distribuição espacial do risco potencial de malária e os padrões espaciais que representam as áreas consideradas de risco para a transmissão da doença, é possivel produzir informação acerca dos fatores ambientais, socioeconômicos e culturais para contribuir com as medidas de controle da malária urbana no município de São João da Baliza.

\section{Os vetores}

Os mosquitos transmissores da malária são insetos da ordem dos dípteros da família Culicidae e do gênero Anopheles. Este gênero compreende cerca de 400 espécies no mundo, das quais, apenas um número reduzido tem importância epidemiológica. No Brasil, cinco espécies são consideradas como vetores principais ou primários. São elas: Anopheles darlingi, Anopheles aquasalis, Anopheles albitarsis, Anopheles cruzi e Anopheles bellator, além de outras espécies consideradas secundárias de menor importância (BRASIL, 2006).

O principal vetor da malária no Brasil é o An. darlingi. Essa espécie frequentemente é encontrada em criadouros formados por água limpa, parada ou de correnteza moderada parcialmente sombreada (Figura 01). O An. aquasalis é um vetor menos importante, porém considerado primário na transmissão da malária, ocorre em regiões costeiras do litoral e tem preferência por criadouros de águas salobras (BRASIL, 2006).

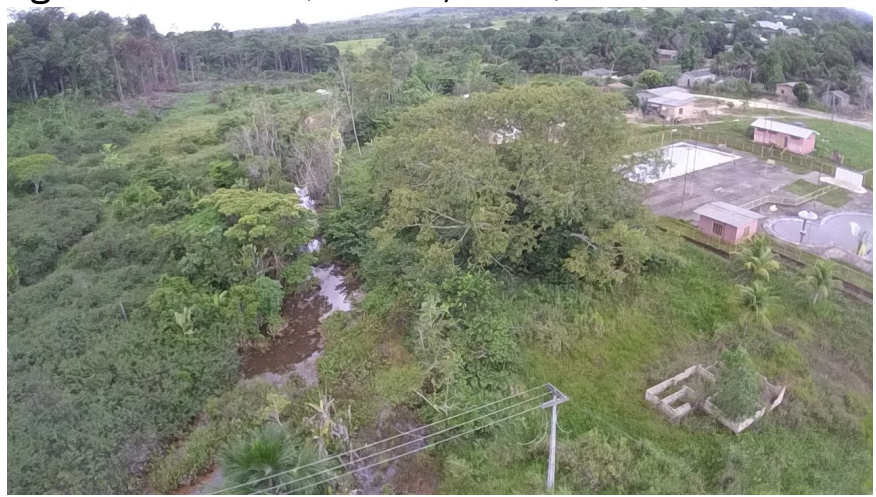

Figura 01 - Criadouro em potencial para Anopheles darlingi na área urbana de São João da Baliza

Fonte: Autor, 2014.

O An. darlingi é, sem sombra de dúvida, o principal transmissor da malária no Brasil. Isso se deve ao comportamento antropofílico desse vetor (preferência por hospedeiros humanos), ao fato de ser altamente susceptivel aos plasmódios humanos e a sua capacidade de transmitir malária dentro e fora das habitações, mesmo em situações de 
baixa densidade. Nas áreas onde An. darlingi está presente, ele é geralmente o anofelino mais frequente dentro do domicílio. É o único anofelino brasileiro encontrado naturalmente infectado pelos três plasmódios que causam malária humana nas Américas, P. vivax, $P$. falciparum e P. malariae e o mais suscetível, experimentalmente, a esses parasitos (CONSOLI e LOURENÇO, 1994).

Outras espécies de anofelinos também são capazes de transmitir malária humana na natureza, contudo, são considerados vetores secundários, principalmente quando coexistem nas mesmas regiões com An. darlingi. São vetores que desenvolvem atividades crepusculares extradomiciliares e demonstram preferência alimentar por hospedeiros não humanos, mas na ausência dos seus hospedeiros preferenciais ou em situações de elevada densidade, podem se alimentar no homem com dada frequência. Em tal caso, tais anofelinos podem, eventualmente, transmitir a malária em áreas onde a endemia fora desencadeada por An. darlingi (CONSOLI e LOURENÇO, 1994).

Para Rosa-Freitas et al. (2010) o habitat preferencial do An. darlingi é a floresta ombrófila densa. Este tipo de floresta está presente mesmo nasecorregiõescuja paisagem é dominada por lavrado, geralmente como floresta aluvial acompanhando as margens dos rios, cruzando a maioria das demais ecorregiões. Estudos recentes conduzidos em Roraima reforçam o papel da floresta aluvial nos padrões de transmissão da malária.

A caracterização e análise de áreas endêmicasparamalária devemconsiderar,além dos fatores ambientais e socioeconômicos, a bioecologia e o comportamento dos vetores envolvidos na transmissão da doença. Estudos sobre a forma de reprodução e atividades vetoriais são fundamentais para o desenvolvimento de ações de vigilância e educação em saúde para auxiliar no controle da incidência de casos da doença.

\section{RESULTADOS E DISCUSSÕES}

\section{Resultados: dados epidemiológicos}

A malária ainda representa um problema de saúde global. Na região das Américas, o Brasil é o País com maior incidência de casos de malária, mais ou menos $50 \%$ dos casos registrados. A região amazônica é responsável pela quase totalidade dos casos de malária que ocorrem no Brasil, cerca de 99,7\%.

De acordo com os dados epidemiológicos, os nove estados da Amazônia legal registraram no período de 2004 a 2014, 3.770 .731 casos de malária. Deste total, 159.736 casos ocorreram no Estado de Roraima, representando 4,23\% do total de casos registrados na região. Os dados também demonstram que no ano de 2005, com exceção do Estado do Maranhão, ocorreu a maior incidência de casos de malária para o período informado na Amazônia. Naquele ano, Roraima registrou o maior número de casos da doença, 30.687 para o período informado.

Quando comparado à variação dos dados de 2014 e 2013, quase todos os estados da Amazônia reduziram a incidência de casos de malária, a exceção foram os estados do Maranhão e de Roraima que registraram aumento de $7,77 \%$ e $13,99 \%$ respectivamente no número de casos da doença. Na variação geral, comparando 2014 e 2013, houve redução de $20,12 \%$ na incidência de casos de malária na Amazônia em 2014.

Considerando os dados do período de 2004 a 2014 apresentados na Tabela 01, a ocorrência de casos de malária no Estado de Roraima foi mais elevada nos municípios de Cantá, Caracaraí e Rorainópolis, com maior incidência para a espécie parasitária vivax. Quando considerado o IPA para a média da série histórica apresentada, $80 \%$ dos municípios de Roraima são considerados de alto risco. O município de Cantá registrou a maior incidência de casos de malária por mil 
habitantes 285,45, a segunda maior incidência parasitária do período informado ocorreu no município de Amajarí, 138,18 casos para mil habitantes. O município de São João da Baliza registrou o maior percentual de casos para o Plasmodium falciparum $24,58 \%$, a forma mais letal da doença.

\begin{tabular}{lccccccc}
\hline \multicolumn{1}{c}{ Municipios } & População & $\begin{array}{c}\text { IPA } \\
\text { (média) }\end{array}$ & $\mathbf{F}$ & $\mathbf{V}$ & $\mathbf{F + V}$ & $\begin{array}{c}\text { Total } \\
\text { Positivo }\end{array}$ & \% F \\
\hline Alto Alegre & 16428 & 55,55 & 877 & 10178 & 53 & 11108 & 8,37 \\
Amajarí & 10432 & 138,18 & 1369 & 10499 & 93 & 11961 & 12,22 \\
Boa vista & 308996 & 2,90 & 791 & 4949 & 43 & 5783 & 14,42 \\
Bonfim & 11525 & 74,6 & 1324 & 8087 & 49 & 9460 & 14,51 \\
Cantá & 15393 & 285,45 & 4983 & 30871 & 349 & 36203 & 14,72 \\
Caracaraí & 19696 & 119,72 & 3200 & 20053 & 189 & 23442 & 14,45 \\
Caroebe & 8826 & 68,08 & 737 & 3868 & 14 & 4619 & 16,25 \\
Iracema & 9762 & 102,09 & 1042 & 6076 & 61 & 7179 & 15,33 \\
Mucajaí & 15890 & 83,5 & 1553 & 9802 & 96 & 11451 & 14,4 \\
Normandia & 9754 & 24,92 & 321 & 1447 & 10 & 1778 & 18,61 \\
Pacaraima & 11423 & 37,57 & 342 & 3432 & 07 & 3781 & 9,23 \\
Rorainópolis & 26326 & 82,7 & 3298 & 18482 & 114 & 21894 & 15,58 \\
São J. da Baliza & 7284 & 69,5 & 1176 & 3638 & 10 & 4824 & 24,58 \\
São L. do Anauá & 7210 & 42,28 & 671 & 2259 & 20 & 2950 & 23,42 \\
Uiramutã & 9127 & 43,81 & 553 & 2794 & 09 & 3356 & 16,74 \\
Total & $\mathbf{4 8 8 0 7 2}$ & $\mathbf{8 2 , 0 5}$ & $\mathbf{2 2 2 3 7}$ & $\mathbf{1 3 6 4 3 5}$ & $\mathbf{1 1 1 7}$ & $\mathbf{1 5 9 7 8 9}$ & $\mathbf{1 4 , 6 1}$ \\
\hline
\end{tabular}

Tabela 01 - Número de casos de malária por municípios de Roraima e espécies parasitárias - 2004 a 2014

Fonte: SIVEP- Malária/SVS/MS, 2015.

Conforme demonstrado no Gráfico 01, a incidência de casos de malária no Estado de Roraima ocorre de forma sazonal. No período compreendido entre os meses de abril a setembro, quando são registrados os maiores índices pluviométricos no estado (período correspondente à estação chuvosa), ocorre uma redução considerável do número de casos de malária. A partir de outubro, após o término do período chuvoso, o número de casos da doença volta a crescer.

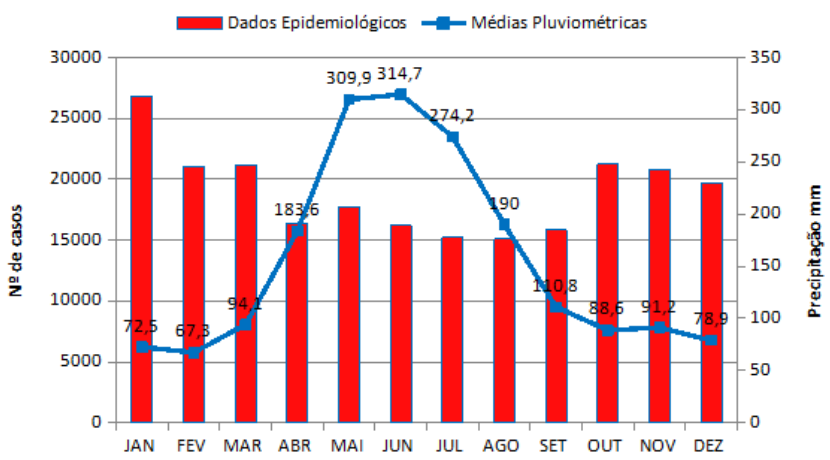

Gráfico 01 - Média mensal de casos de malária (20042014) X médias pluviométricas (2004-2010) - Roraima Fonte: SIVEP-Malária/svs/ms; ANA/SGH, Adaptado por CEPED UFSC, 2011.

Os dados do Gráfico 02 demonstram que de 2004 a 2007, os casos de malária urbana na cidade de São João da Baliza eram inferiores aos registrados na área rural, exceto em 
2005. A partir de 2008 , os números de casos urbanos ultrapassam a ocorrência de casos registrados na área rural, se mantendo em números elevados até 2014. A maior incidência de casos de malária urbana em São João da Baliza ocorreu em 2012, quando foram registrados $92,49 \%$ dos casos da doença na sede do município.

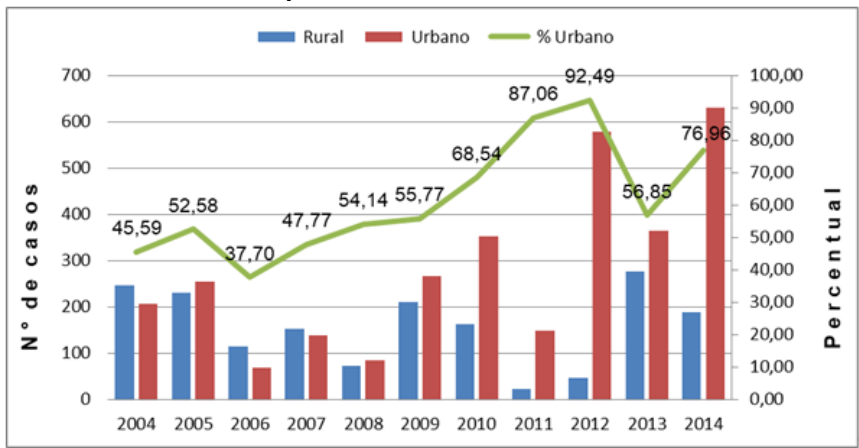

Gráfico 02 - Relação de incidência de casos de Malária Urbana x Rural São João da Baliza - 2004 a 2014

Fonte: SIVEP-Malária/SVS/MS, 2015.

Considerando a ocorrência de casos de malária urbana no município de São João da Baliza do ano de 2014, período que o SIVEPMalária apresentou os dados completos de 12 meses do mesmo ano por bairros, a maior incidência de caos de malária ocorreu no bairro Universo, 29,54\% dos casos da doença. o bairro Centro registrou a segunda maior incidência de casos de malária do perímetro urbano do município, 20,84\%. Ao todo, foram registrados em 2014, 633 casos de malária urbana na cidade de São João da Baliza.

Observando os dados do Gráfico 03, referentes à distribuição de casos de malária por faixa etária no bairro Universo (área de estudos) no ano de 2014, constata-se que o maior número de casos ocorreu nas faixas entre 15 a 49 anos, representando 73,10\% do total de caos da doença. As faixas entre 01 e 14 anos correspondem a cerca de $20 \%$ do número de casos registrados no bairro.

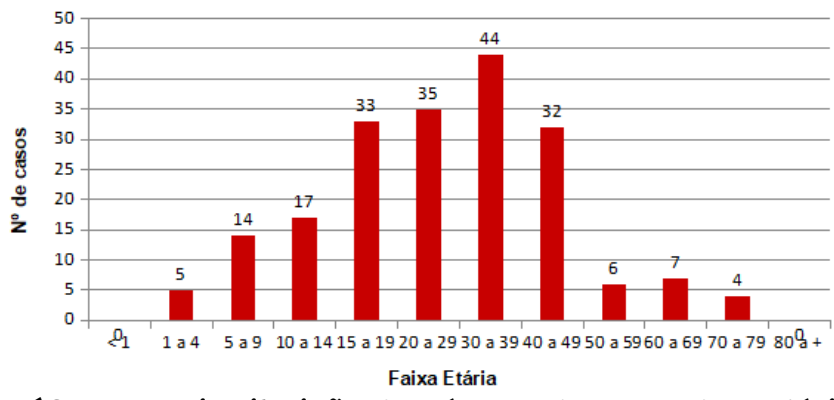

Gráfico 03 - Distribuição do número de casos de malária por faixa etária, Bairro Universo - Ano 2014

Fonte: SIVEP-Malária/SVS/MS, 2015.

\section{Discussão dos resultados}

A análise da informação epidemiológica dos casos de malária disponível nos sistemas de informações oficiais demonstra uma tendência de queda na incidência de casos da doença a partir de 2007 na Região Amazônica no período da série histórica informada. No Estado de Roraima essa redução foi mais significativa a partir de 2011. No ano de 2012, o Estado registrou a maior redução no número de casos de malária, cerca de $50 \%$ em relação ao ano anterior e, no ano de 2013, o menor número de casos da doença 4.826 registros. Para Brasil (2010c) a redução do número de casos de malária se deve principalmente a melhorias na rede de diagnóstico, no tratamento, utilização de medicamentos mais eficazes e a um sistemático monitoramento da situação epidemiológica.

Outro fator que deve ter contribuído para a redução dos casos de malária na Amazônia pode estar relacionado com a redução do desmatamento na região. De 2004 a 2014 houve redução de aproximadamente $80 \%$ do desmatamento na Amazônia brasileira (PRODE/INPE, 2015). Esse fenômeno deve ter contribuído para o declínio dos casos da doença nesse período, já que a incidência de casos de malária na Amazônia tem relação determinante com o processo de desmatamento.

No Estado de Roraima, a distribuição de casos de malária não apresenta um padrão uniforme. Os municípios que concentram a 
maior parte de sua extensão territorial em regiões de Floresta Tropical densa registram o maior número de casos da doença. Nestes municípios, o IPA é de alto risco. Segundo Dias (2003) diversos fatores influenciam a dinâmica de transmissão da malária em regiões de Floresta densa como a Amazônia: Fatores ambientais (vegetação, clima, topografia, hidrografia); socioeconômico (migrações, habitação, renda) e os fatores biológicos (ciclo de vida dos insetos vetores e os agentes parasitários). Para Maciel e Missawa (2012) é fundamental compreender as condições ambientais como fator de favorecimento da ocorrência de criadouros de espécies implicadas na veiculação da doença.

Diversas doenças são delineadas pelos fatores ambientais. Em Roraima, a incidência de casos de malária é sensivelmente influenciada pelos elementos do clima. A redução do número de casos da doença no período da estação chuvosa corrobora com esse entendimento. Para Bustamante (1957 apud Dias, 2003) as chuvas são responsáveis pelo principal fator determinante da periodicidade sazonal da malária, por afetarem a distribuição e densidade dos vetores. Segundo Forattini (1962) isso ocorre por causa do extravasamento dos cursos de água, lavando os criadouros durante a estação chuvosa, causando a morbidade e a diminuição da densidade de determinadas espécies de Anopheles.

O An. (Ny) darlingi, principal vetor da malária na Amazônia, certamente está entre as espécies impactadas pela ação das chuvas, considerando os dados anuais dos registros de casos de malária no Estado de Roraima, percebe-se que há um declínio acentuado do número de casos da doença no período chuvoso, o que reforçaria tal hipótese.

No Estado de Roraima, quando comparado à incidência do número de casos de malária urbana com os números de casos registrados em área rural, os números do município de São João da Baliza são preocupantes. São registrados casos de malária em todos os bairros da sede do município, com o maior número de registros no bairro Universo. A maior ocorrência de casos de malária nesse bairro entre as faixas etárias de 15 e 45 anos pode estar relacionada com atividades laborais. Saraiva (2007) afirma que nessas faixas etárias, os indivíduos são mais ativos economicamente aumentando seu grau de exposição aos vetores da doença em áreas endêmicas. Para Tauil et al. (1985) um dos fatores que favorece a ocorrência de casos de malária está relacionado ao comportamento humano, em regiões de clima quente as pessoas ficam conversando fora dos imóveis a partir do crepúsculo vespertino, expondo-se aos vetores da malária.

Os dados epidemiológicos confirmam que o bairro Universo é uma localidade de alto risco para a transmissão de malária. Para Brasil (2010d) os criadouros preferenciais de An. (Ny) darlingi principal vetor da malária são coleções de água limpa, quente, sombreada e de baixo fluxo, muito frequentes na Amazônia brasileira. Essas características foram identificadas no espaço geográfico do bairro Universo.

Braz, Duarte e Tauil (2013) reiteram que a ocorrência de casos de malária está relacionada com as formas de uso da terra, o comportamento humano, os elementos físicos e biológicos do meio natural e as transformações nele causadas pela intervenção antrópica. Neste sentido, a análise dos dados sugere que os fatores ambientais e socioeconômicos exercem uma relação determinante para a ocorrência de caos de malária urbana no bairro Universo em São João da Baliza.

A compreensão desses fatores enquanto agentes de interação ou facilitadores da ocorrência de agravos como a malária, é fundamental para o planejamento de políticas mais eficazes de prevenção e controle da doença no bairro Universo. Tauil et al. (1985) afirmam que o An. (Ny) darlingi, não se reproduz 
em coleções hídricas com margens livres de vegetação. Assim, é possivel controlar o vetor apenas podando a vegetação responsável pelo sombreamento do lugar de reprodução do vetor.

Considerando a análise dos dados e dos referenciais utilizados, é possivel sugerir como medidas mais efetivas para o controle da malária no bairro Universo, além do diagnóstico precoce e o tratamento oportuno, o manejo ambiental dos sítios de reprodução dos vetores (clareamento dos cursos naturais de água através da poda da vegetação localizada nas margens, a remoção de detritos para desobstrução do leito dos igarapés), ações de educação em saúde abordando os mecanismos de produção da doença e as formas de prevenção individuais e coletivas. Essas ações são mais indicadas para áreas urbanas em decorrência da limitação e a baixa efetividade das ações de controle químico.

As ações de educação em saúde no âmbito do controle de endemias têm como principal objetivo, promover consciência crítica na sociedade a respeito de problemas gerados por agravos como, por exemplo, a malária, possibilitando o planejamento e a execução de ações considerando a realidade local. Quanto mais informada estiver a população, maior será a possibilidade de participar ativamente dos esforços para 0 controle da doença.

\section{CONSIDERAÇÕES FINAIS}

Os altos índices de casos de malária registrados na área urbana de São João da Baliza foram determinantes para a realização deste trabalho. Como área de estudo, utilizouse o bairro Universo por este concentrar $29,47 \%$ dos casos de malária registrados na sede do município, o que correspondeu ao IPA de 264 casos de malária para cada 1.000 habitantes em 2014. Verificou-se que essa localidade possui uma rica bacia hidrográfica formada por igarapés, que são o principal sítio de reprodução do An. darlingi, principal vetor da malária na Amazônia.

Neste contexto, foi possivel constatar que os elementos ambientais (coleções hídricas formadas por igarapés, vegetação, temperatura e umidade) e os elementos socioeconômicos (localização dos imóveis, densidade populacional em áreas de risco, fluxo migratório entre as áreas urbana e rural) são fatores que exercem forte influência sobre a dinâmica espacial de transmissão da malária no bairro Universo.

Portanto, considerando a análise dos dados produzidos pela pesquisa, os resultados sugerem como medidas mais efetivas para o controle da malária, além do diagnóstico precoce e o tratamento oportuno, o manejo ambiental dos sítios de reprodução dos vetores (clareamento dos cursos naturais de água através da poda da vegetação localizada nas margens, a remoção de detritos para desobstrução do leito dos igarapés), ações de educação em saúde abordando os mecanismos de produção da doença e as formas de prevenção individual e coletiva.

Por fim, essas ações são mais indicadas para áreas urbanas em decorrência da limitação e a baixa efetividade das ações de controle químico. Cabe ressaltar que a dinâmica espacial de transmissão da malária na área urbana de São João da Baliza é análoga, podendo ser adotado as mesmas ações para todos os bairros.

\section{REFERÊNCIAS}

AB'SABER, A. N. Um Conceito de Geomorfologia a Serviço das Pesquisas sobre o Quaternário. Geomorfologia (18). São Paulo: IGUSP, 1969.

BENI, M. C. Análise estrutural do turismo. São Paulo: Senac, 1998.

BERTRAND, G. Paisagem e Geografia Física Global - esboço metodológico. Cadernos de 
Ciências da Terra. (13). São Paulo: IGUSP, 1971.

BOUllón, R. C. Planejamento do espaço turístico. Bauru: EDUSC, 2002.

DEAN, Warren. A ferro e fogo: a história e a devastação da Mata Atlântica brasileira. São Paulo: Companhia das Letras, 2013.

EMPRESA DAS ARTES. Tourist Guide Roraima: Ecology, History and Culture. São Paulo, 2009.

IBGE/Instituto Brasileiro de Geografia e Estatística. Dados Censitários. Rio de Janeiro, 2015.

HOLANDA, Lêda R.; MARMOS, José Luiz; MAIA, Maria Adelaide. (Orgs.) Geodiversidade do Estado de Roraima. Manaus: CPRM, 2014.

LADEIRA, L.F.B.;DANTAS,M.E.Compartimentação Geomorfológica. In HOLANDA, Janólfta L. R.; MARMOS, José Luiz; MAIA, Adelaide M.. (Orgs.). Geodiversidade do Estado de Roraima. Manaus: CPRM, 2014. (31-46).

LENCIONI, S. Região e Geografia. São Paulo: EDUSP, 1999.

MARION, N. M. \& FARELL, B. C. A. tale of tourism in two cities. Annals of Tourism Research, 20 (336-353). London, 1998.

RÖLKE, P. Wander \& Naturfüher: Sächsiche Schweis. Band 1. Dresden: Berg- \& Naturverlag Rölke, 2006.

Wander \& Naturfüher: Sächsiche

Schweis. Band 2. Dresden: Berg \& Naturverlag Rölke, 2006.

ROSS, J. L. S. Análise e Síntese na Abordagem Geográfica da Pesquisa para Planejamento Ambiental. Revista do Departamento de Geografia, № 9. FFLCH/USP, São Paulo, 1995.

SEABRA, Giovanni F. Turismo de Base Local no Nordeste Brasileiro. In NASCIMENTO, Ângela; LIMA, Marcos Costa. (orgs). 0 Nordeste Brasileiro em questão: uma agenda para reflexão 2. (389-418). Recife: Sudene, 2016.

Paraíba. João Pessoa: Editora Universitária / UFPB, 2014.

.Turismo Sertanejo. João Pessoa:

Editora Universitária / UFPB, 2007a.

Potencial Geoturístico no Nordeste Brasileiro. Caminho das Pedras: a paisagem sertaneja e o lugar do turismo. In Anais do I Simpósio de Geografia Física do Nordeste. Universidade Regional do Cariri. Crato, 2007b.

. Caminho das Pedras. O turismo ecológico de base geológica no Agreste Pernambucano. Relatório de Pós-Doutorado. Recife: Departamento de Geologia / CTG / UFPE, 2004.

Ecos do Turismo: o turismo ecológico em áreas protegidas. Campinas: Papirus, 2001a.

Pesquisa Científica: o método em questão. Brasília:Editora UNB, 2001b.

Do Garimpo aos Ecos do Turismo: o Parque Nacional da Chapada Diamantina. Tese de Doutorado. São Paulo: FFLCH / USP, 1998.

Estudo Geomorfológico da Região Cárstica de Andaraí: uma contribuição à conservação de cavernas. Dissertação de Mestrado em Geografia Física. Recife: CFCH / UFPE, 1991.

SEABRA, G. F.; Neu, C. O turismo no espaço rural da Chapada Diamantina. Anais do CITURDES - IV Congresso Internacional sobre Turismo Rural e Desenvolvimento Sustentável, Joinville, 2004. 
SCHOBBENHAUS, C.; SILVA, C. R. (Orgs.). Geoparques do Brasil: Propostas. Vol. 1. Rio de Janeiro: CPRM, 2012.

SOTCHAVA, V. B. O Estudo dos Geossistemas. Métodos em Questão. (16). São Paulo: IGUSP, 1972.

TIMOTHY, D. J. Cooperative tourism planning in a developing destination. Journal of sustainable tourism. V. 6, n. 1. London, 1998. 